\title{
Sleep spindles are resilient to extensive white matter deterioration
}

\author{
Erlan Sanchez, 1,2 Caroline Arbour, ',3 Héjar El-Khatib,, 1,4 Karine Marcotte, ',5 Hélène Blais,' \\ Andrée-Ann Baril, ${ }^{6}$ Christophe Bedetti, ${ }^{4}$ Maxime Descoteaux, ${ }^{7}$ Jean-Marc Lina, ${ }^{1,8}$ \\ Danielle Gilbert,' Julie Carrier ${ }^{1,4}$ and Nadia Gosselin ${ }^{1,4}$
}

Sleep spindles are an essential part of non-rapid eye movement sleep, notably involved in sleep consolidation, cognition, learning and memory. These oscillatory waves depend on an interaction loop between the thalamus and the cortex, which relies on a structural backbone of thalamo-cortical white matter tracts. It is still largely unknown if the brain can properly produce sleep spindles when it underwent extensive white matter deterioration in these tracts, and we hypothesized that it would affect sleep spindle generation and morphology. We tested this hypothesis with chronic moderate to severe traumatic brain injury $(n=23$; $30.5 \pm 11.1$ years old; $17 \mathrm{~m} / 6 \mathrm{f}$ ), a unique human model of extensive white matter deterioration, and a healthy control group ( $n=27 ; 30.3 \pm 13.4$ years old; $21 \mathrm{~m} / 6 \mathrm{f}$ ). Sleep spindles were analysed on a full night of polysomnography over the frontal, central and parietal brain regions, and we measured their density, morphology and sigma-band power. White matter deterioration was quantified using diffusion-weighted MRI, with which we performed both whole-brain voxel-wise analysis (Tract-Based Spatial Statistics) and probabilistic tractography (with High Angular Resolution Diffusion Imaging) to target the thalamo-cortical tracts. Group differences were assessed for all variables and correlations were performed separately in each group, corrected for age and multiple comparisons. Surprisingly, although extensive white matter damage across the brain including all thalamo-cortical tracts was evident in the brain-injured group, sleep spindles remained completely undisrupted when compared to a healthy control group. In addition, almost all sleep spindle characteristics were not associated with the degree of white matter deterioration in the braininjured group, except that more white matter deterioration correlated with lower spindle frequency over the frontal regions. This study highlights the resilience of sleep spindles to the deterioration of all white matter tracts critical to their existence, as they conserve normal density during non-rapid eye movement sleep with mostly unaltered morphology. We show that even with such a severe traumatic event, the brain has the ability to adapt or to withstand alterations in order to conserve normal sleep spindles.

1 Research Center of the Centre Intégré Universitaire de Santé et de Services Sociaux du Nord de l'Île-de-Montréal, Montreal H4J 1C5, Canada

2 Department of Neuroscience, Université de Montréal, Montreal H3T 1J4, Canada

3 Faculty of Nursing, Université de Montréal, Montreal H3T 1A8, Canada

4 Department of Psychology, Université de Montréal, Montreal H2V 2S9, Canada

5 School of Speech Language Pathology and Audiology, Université de Montréal, Montreal H3N 1X7, Canada

6 The Framingham Heart Study, Boston University School of Medicine, Boston 02118, USA

7 Computer Science Department, Universite de Sherbrooke, Sherbrooke J1K 2R1, Canada

8 Department of electrical engineering, École de Technologie Supérieure, Montreal H3C 1K3, Canada

Correspondence to: Nadia Gosselin, PhD

Research Center of the Centre Intégré Universitaire de Santé et de Services Sociaux du Nord de l'île-de-Montréal Montreal H4J 1C5, Canada

E-mail: nadia.gosselin@umontreal.ca

Received February 13, 2020. Revised April 21, 2020. Accepted April 27, 2020. Advance Access publication June 13,2020 (C) The Author(s) (2020). Published by Oxford University Press on behalf of the Guarantors of Brain.

This is an Open Access article distributed under the terms of the Creative Commons Attribution Non-Commercial License (http://creativecommons.org/licenses/by-nc/4.0/), which permits non-commercial re-use, distribution, and reproduction in any medium, provided the original work is properly cited. For commercial re-use, please contact journals.permissions@oup.com 
Keywords: sleep; spindles; white matter; TBI; traumatic brain injury

Abbreviation: $\mathrm{TBI}=$ traumatic brain injury

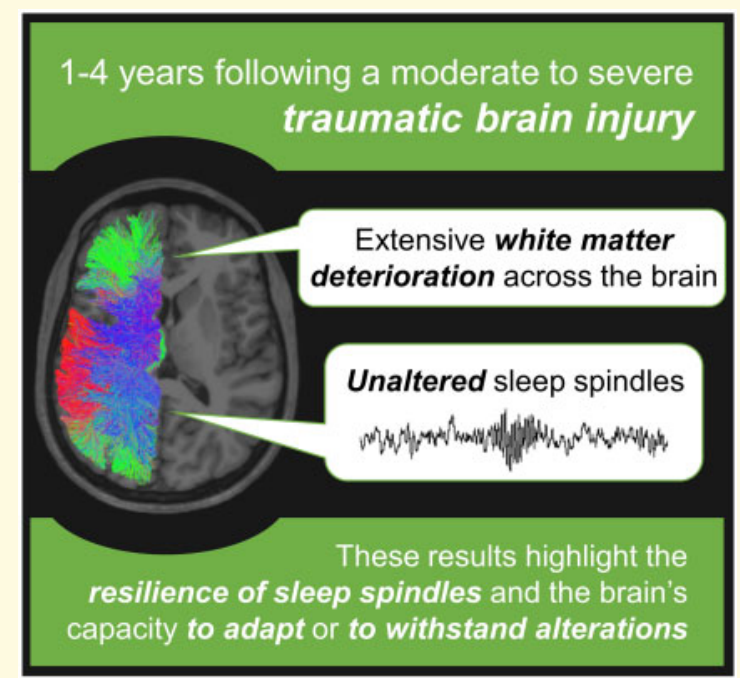

\section{Introduction}

Sleep spindles are oscillatory events that occur during non-rapid eye movement sleep and consist in rapid bursts of $11-16 \mathrm{~Hz}$ detectable on the EEG. They are involved in learning and memory (Gais et al., 2002; Schabus et al., 2006; Fogel and Smith, 2011), in global cognitive aptitude (Schabus et al., 2006; Fogel et al., 2007; Fogel and Smith, 2011) and in preventing sleep-interrupting arousals (Dang-Vu et al., 2011; Schabus et al., 2012). Since their discovery, numerous efforts have been deployed to identify factors determining sleep spindle characteristics. Understanding how spindles are impacted by normal or pathological brain physiology could improve our knowledge about inter-individual differences in the ability to learn and memorize.

Recent studies have pointed to white matter inter-individual variations to explain sleep spindle characteristics, the rationale being that they depend on the complex interaction between the thalamus and the cortex, in which cortico-thalamic neurons, thalamo-cortical neurons and reticular neurons of the thalamus tightly interact (Clawson et al., 2016). Variations in the structural backbone of this thalamo-cortical loop could impact spindle generation, amplitude, as well as oscillation frequency, and therefore their role in cognitive functioning. A first study found that higher axial diffusivity in the corpus callosum, temporal fascicles and tracts surrounding the thalamus, interpreted in this case as a marker of better white matter integrity, was associated with higher spindle density and sigma-band power in young healthy adults (Piantoni et al., 2013). Two other studies investigated how white matter moderates the relationship between spindles and motor memory consolidation, and found that its integrity strengthens this association (Mander et al., 2017; Vien et al., 2019). Finally, one recent study found that markers of better thalamo-frontal white matter integrity predict higher spindle amplitude and sigma-band power in young individuals only, and not in older adults (Gaudreault et al., 2018). However, as these studies only included healthy young or old adults, the range of white matter integrity examined mainly consisted in the small non-pathological inter-individual variations expected in normal development. Having greater variability in white matter would more clearly identify its impact on spindle characteristics.

Widespread and significant white matter damage is the signature of chronic moderate to severe traumatic brain injury (TBI) (Johnson et al., 2013). Cognitive and functional outcomes are extremely variable after moderate to severe TBI, and this variability is also found in the extent of white matter damage (Kraus et al., 2007; Kennedy et al., 2009; Kumar et al., 2009; Haberg et al., 2015; Sanchez et al., 2019). Importantly, thalamo-cortical tracts are especially vulnerable to TBI due to the head rotational acceleration during the initial injury, resulting in shear and tensile damage leading to delayed secondary disconnection (Gennarelli et al., 1982; Johnson et al., 2013; Smith et al., 2013). Regarding sleep spindles in adults with moderate to severe TBI, only one preliminary study has examined eight TBI participants with good outcomes during a 30 -min nap at 151 days postinjury and no difference in spindle morphology was found when compared with healthy controls, although decreases in amplitude and frequency were observed at 80 days 
post-injury (Urakami, 2012). Therefore, it is still mostly unknown whether the brain can properly produce sleep spindles when it underwent extensive white matter deterioration in white matter tracts critical to their generation. Thus, the aim of this study was to characterize the association between sleep spindles and thalamo-cortical white matter damage using a moderate to severe TBI model.

We tested our participants with diffusion-weighted brain MRI sequences, using probabilistic tractography to reconstruct thalamo-cortical tracts in addition to a whole-brain voxel-wise approach. We inferred white matter integrity by measuring water diffusion properties in the white matter tracts. Participants with chronic TBI are expected to present a large range of white matter deterioration (Kraus et al., 2007; Kennedy et al., 2009; Kumar et al., 2009; Pitkonen et al., 2012; Haberg et al., 2015; Sanchez et al., 2019). All participants then underwent a full night of polysomnography, in which we measured spindle density, amplitude, duration and oscillation frequency, as well as sigma-band power. Considering previous findings in healthy populations where the integrity of thalamo-cortical tracts was associated with spindle density and amplitude (Piantoni et al., 2013; Mander et al., 2017; Gaudreault et al., 2018; Vien et al., 2019), we hypothesized that more white matter damage, globally and in the thalamo-cortical tracts, would affect spindles, resulting in lower density, lower sigma-band power and spindles of lower amplitude. Due to the effect of white matter damage on axonal conduction speed (Waxman, 1977; Hartline and Colman, 2007; Marion et al., 2018), we also hypothesized that white matter deterioration would result in slower spindle oscillation frequency, which represents the speed at which the impulses complete the loop between the thalamus to the cortex.

\section{Material and methods}

\section{Participants}

Twenty-three participants aged between 18 and 56 years old (mean: $30.5 \pm 11.1$ years old; $17 \mathrm{~m} / 6 \mathrm{f}$ ) and diagnosed with first-time moderate to severe TBI were recruited for this study. They were all treated at a tertiary trauma centre (Hôpital du Sacré-Coeur de Montréal) in the acute phase of their injury. Injury-related data were collected from their hospital charts. At the time of testing, participants were in the chronic phase of their injury, at least 11 months and up to 39 months following the trauma. TBI is defined as an alteration in brain function, or other evidence of brain pathology, caused by an external force (Menon et al., 2010). Diagnosis of moderate or severe TBI was given by a neurosurgeon expert in TBI according to standard criteria (Teasdale and Jennett, 1974), including a Glasgow Coma Scale score between 3 and 12 and a posttraumatic amnesia of at least $24 \mathrm{~h}$ (Nakase-Richardson et al., 2009). Twenty-seven healthy controls were also included in this study and were of similar age and sex than TBI participants. Relevant data on demographic and clinical characteristics have been more thoroughly detailed in our previous work (Sanchez et al., 2019). For all participants, exclusion criteria were: (i) history of multiple TBI (for patients) or previous TBI (for healthy controls), (ii) quadriplegia, (iii) regular intake of sleep medication, (iv) history of psychiatric, neurologic or substance use disorders, (v) obesity (body-mass index $>30 \mathrm{~kg} / \mathrm{m}^{2}$ ), (vi) recent trans-meridian trip or night shift work and (vii) MRI contraindications, often in the form of metallic implants left by surgeries. The study was approved by the Centre Intégré Universitaire de Santé et de Services Sociaux du Nord de l'Île-de-Montréal Research Ethics Board and written consent was obtained from each participant, compliant with the Declaration of Helsinki. These participants' polysomnography and MRI data have been included in our previous work (Sanchez et al., 2019).

\section{Polysomnography recording}

Bedtime and wake time for the in-laboratory recording were chosen in accordance with the participant's normal sleep schedule, determined by a week of sleep diaries and activity monitoring using an actimetric device (ActiwatchL or Actiwatch-Spectrum, Philips Healthcare, Andover, MA) during the week preceding the visit at the laboratory. The full night of polysomnography recording included EEG, electrooculogram, chin and tibia electromyograms, and electrocardiogram. The following EEG derivations were acquired: FP1, FP2, Fz, F3, F4, F7, F8, $\mathrm{Cz}, \mathrm{C} 3, \mathrm{C} 4, \mathrm{Pz}, \mathrm{P} 3, \mathrm{P} 4, \mathrm{O} 1, \mathrm{O} 2, \mathrm{~T} 3, \mathrm{~T} 4, \mathrm{~T} 5, \mathrm{~T} 6$. The acquired signals were digitalized with a commercial acquisition software (Harmonie Stellate Systems, Montreal, Canada). The standard criteria from the American Academy of Sleep Medicine manual were used for scoring sleep stages and events (Iber et al., 2007). Artefacts were marked by both automatic and visual detection, and the artefacted epochs were excluded from further analysis.

\section{Spindle detection and sigma spectral analysis}

We detected spindles occurring during the N2 and N3 stages of non-rapid eye movement sleep for all sleep cycles by using an automatic algorithm (Martin et al., 2013) on artefact-free epochs. Detection was performed on selected frontal, central and parietal electrodes (F3, F4, Fz, C3, C4, Cz, P3, P4, Pz) for the entire night. The raw EEG signal was first band pass filtered $(11-14.9 \mathrm{~Hz})$ using a linear phase finite impulse response filter $(-3$ $\mathrm{dB}$ ), and the root-mean-square amplitude of the filtered signal was then calculated over $0.25 \mathrm{~s}$ epochs. The threshold for spindle detection was set at the 95th percentile and at a minimum duration of $0.5 \mathrm{~s}$. 
In addition to measuring spindle density (events per minute), we also identified the morphological characteristics of every spindle detected: largest peak-to-peak amplitude $(\mu \mathrm{V})$, frequency $(\mathrm{Hz})$ and duration $(\mathrm{s})$. To quantify sigma activity power, a Fast Fourier Transform (extended cosine bell tapering, $5 \mathrm{~s}$ artefact-free epochs) was carried out on $\mathrm{N} 2$ and N3 stages of non-rapid eye movement sleep, for the entire night. Absolute power was calculated for the sigma frequency band $(11-16 \mathrm{~Hz})$.

\section{MRI acquisition and pre-processing}

MRI data were acquired using a 3T Siemens Magnetom Trio scanner at the Unite de Neuroimagerie Fonctionnelle of the Institut Universitaire de Gériatrie de Montreal, in the afternoon preceding the polysomnographic recording. All participants underwent a diffusion-weighted MRI sequence (with additional gradient field maps and AP/PA b0 sequences) and a $\mathrm{T}_{1}$-weighted sequence. The diffusion parameters were as follows: echo-planar imaging, 64 non-collinear directions, image resolution $=2 \mathrm{~mm}^{3}, 72$ slices, repetition time $=9500 \mathrm{~ms}$, echo time $=93 \mathrm{~ms}, b$-value $=1000 \mathrm{~s} /$ $\mathrm{mm}^{2}$, duration $=648 \mathrm{~s}$. The $\mathrm{T}_{1}$ parameters were as follows: image resolution $=1 \mathrm{~mm}^{3}$, repetition time $=2530$ $\mathrm{ms}$, echo time $=1.64 \mathrm{~ms}$, duration $=363 \mathrm{~s}$. All MRI were inspected by a neuroradiologist prior to inclusion in the study data to detect large anomalies that may cause exclusion (i.e. specific brain lesions that could affect our analyses).

Diffusion data pre-processing was performed with the standard pipeline from the Toolkit for Analysis in Diffusion MRI (https://unf-montreal.ca/mri-data-process ing/; last accessed 10 June 2020). More specifically, $T_{1}$ data were first parcellated with the Freesurfer (https://surf er.nmr.mgh.harvard.edu/; last accessed 10 June 2020) recon-all pipeline v6.0.0 using CBRAIN (https://cbrain.ca/; last accessed 10 June 2020), then diffusion data were denoised, corrected for motion and distortions, upsampled at $1 \mathrm{~mm}^{3}$ resolution and registered with the parcelled anatomical images and atlases.

\section{Tract-based spatial statistics}

For voxel-wise analysis, FSL diffusion tensors were fitted over each voxel, and tensor metrics (fractional anisotropy; mean, axial and radial diffusivities) were computed. To allow for accurate group analyses, diffusion data were prepared using Tract-Based Spatial Statistics (https://fsl.fmrib. ox.ac.uk/fsl/fslwiki/TBSS/; last accessed 10 June 2020) (Smith et al., 2004, 2006). We started by affine-aligning the fractional anisotropy data to the standard 1-mm MNI152 brain. This was performed using FNIRT, a nonlinear registration tool. Then, a skeleton of the average white matter tracts was created by averaging and thresholding $(>0.2)$ all participants' fractional anisotropy data. Then, each participant's fractional anisotropy as well as mean, axial and radial diffusivity data were projected onto this skeleton to produce the final images used as input for voxel-wise analyses.

\section{Probabilistic tractography}

For probabilistic tractography, DTI and high angular resolution diffusion imaging metrics were computed with the DIPY software (https://dipy.org/; last accessed 10 June 2020) (Garyfallidis et al., 2014). Fibre orientation distribution functions were estimated using non-negativity constrained spherical deconvolution (Descoteaux et al., 2009). For each subject, the whole-brain structural connectome was computed using the MRtrix (http://www.mrtrix.org/; last accessed 10 June 2020) probabilistic tractography algorithms (tckgen, tcksift and tck2connectome) integrated into the Toolkit for Analysis in Diffusion MRI pipeline. The default parameters were used: 1 million streamlines generated with a step size of $2 \mathrm{~mm}$ and a maximum length of $300 \mathrm{~mm}$. The thalamocortical tracts were then extracted with the tract_querier software (https://github.com/demianw/tract_querier/; last accessed 10 June 2020) using the White Matter Query Language (Wassermann et al., 2013, 2016). We targeted seven thalamo-cortical bundles for each hemisphere (i.e. thalamo-prefrontal, thalamo-premotor, thalamo-precentral, thalamo-postcentral, thalamo-parietal, thalamo-cortical) using logic functions and Freesurfer's anatomical atlases. These queries are described in Table 1, based on previously defined projection tracts (Wassermann et al., 2016). A filtering algorithm was then used to remove outliers from the extracted tracts. Finally, tensor metrics (fractional anisotropy, mean, axial and radial diffusivity) and tract volume were computed for each bundle, and the mean bundle values for each subject were used in further analyses.

\section{Statistical analyses}

The TBI and control groups were compared for demographic, sleep macro-architecture and sleep spindle characteristics using two-tailed Student's $t$-tests or Chi-square. We formed three clusters with frontal (F3, F4, Fz), central $(\mathrm{C} 3, \mathrm{C} 4, \mathrm{Cz})$ and parietal $(\mathrm{P} 3, \mathrm{P} 4, \mathrm{Pz})$ electrodes where detection occurred, in which spindle characteristics were averaged for each subject prior to statistical analyses. Pooling electrodes allowed us to retain four participants for which one to two electrodes were rejected after artefact detection. To assess global group differences in thalamocortical white matter bundles, we performed two-tailed $t$ tests for each cortical bundle extracted. All these statistical analyses were performed on SPSS Statistics 25 (IBM Corp., 2017), with statistical significance set at $P<0.01$.

Voxel-wise correlational statistics on processed white matter images were carried using Randomise (https://fsl.fmrib. ox.ac.uk/fsl/fslwiki/Randomise/; last accessed 10 June 2020) (Winkler et al., 2014). This technique uses Conditional Monte Carlo permutations to test standard General Linear Model statistics. We used 10000 permutations per contrast, resulting in a confidence limit of \pm 0.0044 for 
Table I Queries for each thalamo-cortical fibre bundle

\begin{tabular}{|c|c|}
\hline Projection fibre bundle & Command \\
\hline Thalamo-prefrontal & Thal-pref.side $=$ (endpoints_in(thalamus.side) and endpoints_in(prefrontal.side) $)$ \\
\hline Thalamo-premotor & Thal-prem.side $=($ endpoints_in(thalamus.side) and endpoints_in(premotor.side) $)$ \\
\hline Thalamo-precentral & Thal-prec.side = (endpoints_in(thalamus.side) and endpoints_in(precentral.side) $)$ \\
\hline Thalamo-postcentral & Thal-postc.side $=($ endpoints_in(thalamus.side) and endpoints_in(postcentral.side) $)$ \\
\hline Thalamo-parietal & Thal-par.side $=($ endpoints_in(thalamus.side $)$ and endpoints_in(parietal.side) $)$ \\
\hline \multirow[t]{3}{*}{ Thalamo-cortical } & Thal-cor.side = (endpoints_in(thalamus.side) and (endpoints_in(prefrontal.side) \\
\hline & or endpoints_in(premotor.side) or endpoints_in(precentral.side) or \\
\hline & endpoints_in(postcentral.side) or endpoints_in(parietal.side))) \\
\hline
\end{tabular}

$P=0.05$. Threshold-Free Cluster Enhancement was also used to enhance cluster-like structures while retaining the voxel-wise nature of the images; this is done by taking into account the cluster-like local spatial support of each voxel. We assessed the voxel-wise association between white matter diffusion parameters (fractional anisotropy, mean, axial and radial diffusivity) and spindle variables (density, amplitude, frequency, duration, sigma activity power). These analyses were performed separately on the TBI and control groups for each cluster of electrodes, with age as a confound regressor and family-wise error correction. The ICBM-DTI-81 white matter atlas was used to identify significant results (Mori et al., 2008).

To investigate the association between thalamo-cortical white matter and spindles variables in TBI compared to the control group, we performed hierarchical linear regressions. We tested whether groups moderate the association between independent variables (average fractional anisotropy, mean, axial and radial diffusivity for each extracted white matter bundle) and dependant variables (spindle density, amplitude, frequency, duration and sigma activity power, for each electrode cluster). Age was included as a control variable in Step 1 of the model because of its strong association with sleep and white matter structure. Step 2 included the independent variable and groups, and Step 3 included the independent $\mathrm{X}$ groups interaction variable. Post hoc Pearson's correlations were conducted when significant interactions were found. Statistical significance was set at $P<0.01$ to correct for type I errors.

\section{Data availabillity}

Relevant data that support the findings of this study are available from the corresponding author upon request.

\section{Results}

\section{Participant characteristics}

Demographic and clinical characteristics, as well as sleep macro-architecture for both groups have been thoroughly detailed in our previously published work (Sanchez et al., 2019). Briefly, the TBI group $(n=23$; $30.5 \pm 11.1$ years old; $17 \mathrm{~m} / 6 \mathrm{f}$ ) was of similar age and sex than the healthy control group $(n=27$;
$30.3 \pm 13.4$ years old; $21 \mathrm{~m} / 6 \mathrm{f}$ ), and comprised only individuals diagnosed with a moderate to severe TBI (Glasgow Coma Scale $=8.5 \pm 3.3$; Post-traumatic amnesia duration $=17.0 \pm 17.6)$. All TBI participants were in the chronic phase, at least 11 months and up to 39 months following the injury (average of $23.4 \pm 9.4$ months). The TBI group reported worse sleep quality and more fatigue compared to healthy controls but had normal sleep macro-architecture when measured with polysomnography. Indeed, there was no significant group difference in sleep latency, total sleep time, wake after sleep onset duration, sleep efficiency, sleep stage duration or apnoea-hypopnoea index. There was also no group difference in total sleep time for the week preceding testing when measured with sleep diaries and actimetry.

In terms of white matter, TBI subjects showed a very large range of damage over most brain areas when compared to the control group using Tract-Based Spatial Statistics (Fig. 1a). In regions where group differences were found for fractional anisotropy, all except three TBI subjects presented a fractional anisotropy reduction ranging from one to almost seven standard deviations below the control group mean (Fig. 1b). A clear group difference was also observed when restricting white matter to thalamo-cortical tracts using high angular resolution diffusion imaging probabilistic tractography (Fig. 1c and d). By using two-tailed $t$-tests to compare groups on each thalamo-cortical tracts as well as for the whole thalamocortical bundle (see Table 1), we found that TBI subjects had significantly lower fractional anisotropy and higher diffusivities when compared with control subjects in all tracts (Fig. 1d), suggesting clinically significant axonal and myelin deterioration. In our sample, time from TBI did not correlate with either global white matter metrics or spindle characteristics.

\section{Sleep spindles remain unchanged in TBI versus controls}

Sleep spindle characteristics detected on frontal, central and parietal electrodes in a full night of polysomnography were compared between TBI and control groups using two-tailed $t$-tests, in each electrode cluster. No significant group difference was found for spindle density, 

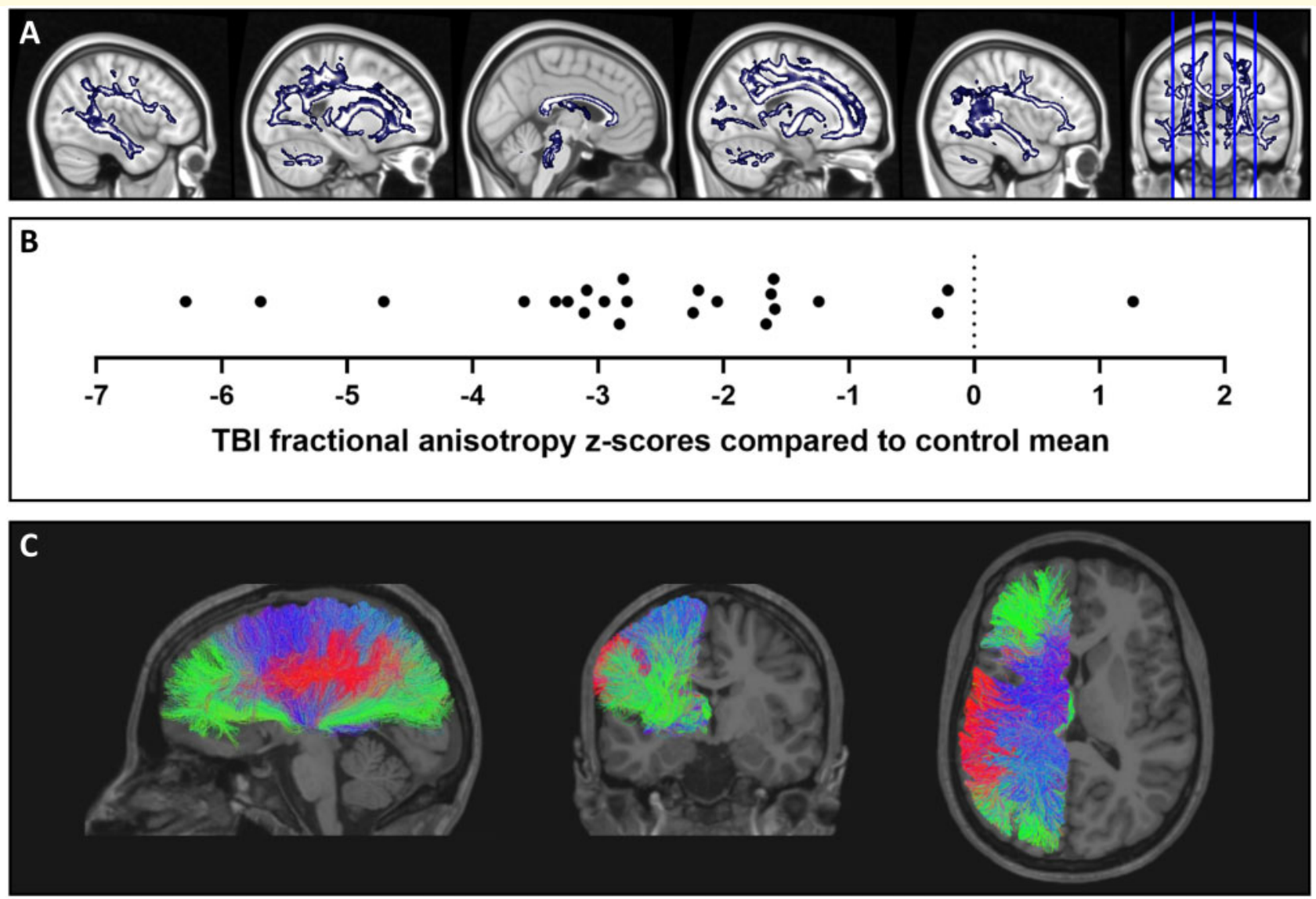

D
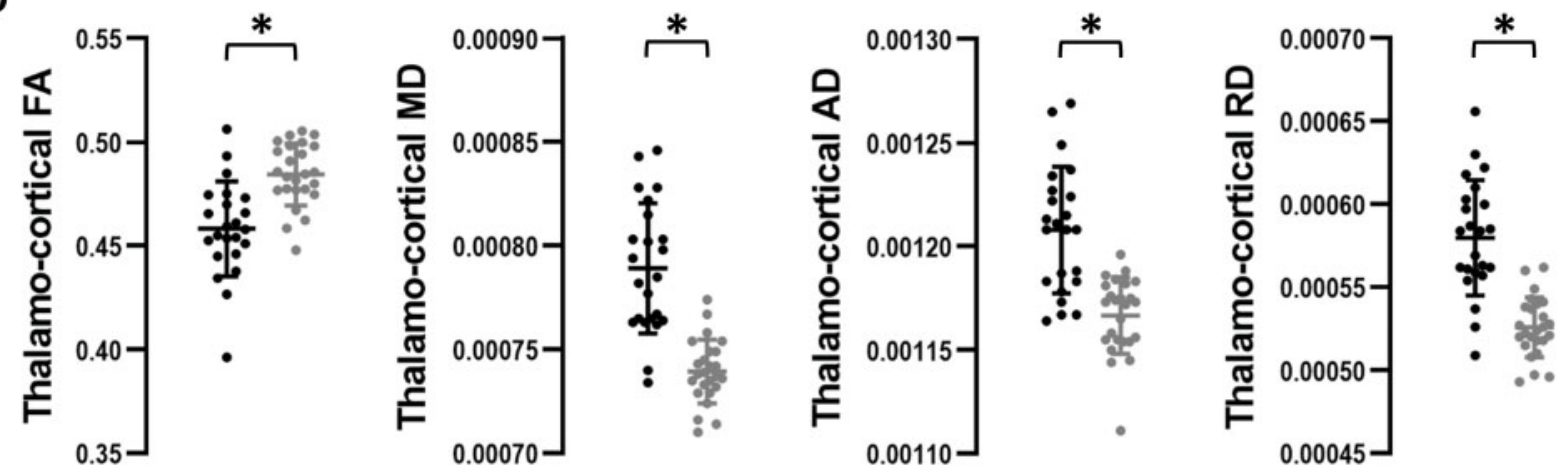

${ }^{*} \mathrm{p}<0.0001$
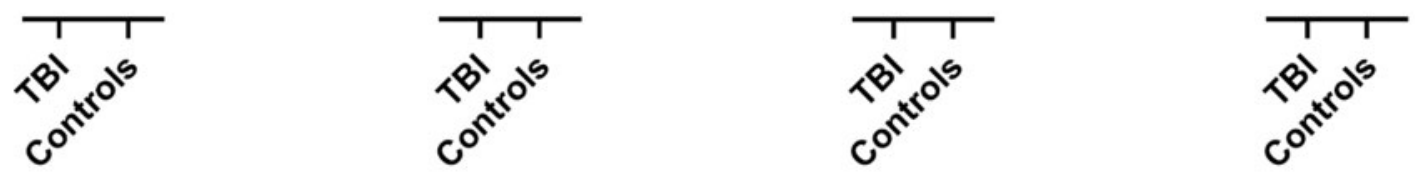

Figure I White matter deterioration in chronic moderate to severe TBI compared to controls. (a) Brain regions where fractional anisotropy was significantly lower in the TBI group when compared to the control group with Tract-Based Spatial Statistics voxel-wise $t$-tests (thresholded at $P<0.05$, corrected for age and multiple comparisons). Results are overlaid over the MNII $52 \mathrm{TI} I \mathrm{~mm}$ brain. (b) TBI z-scores, calculated from the mean fractional anisotropy values for each TBI subject compared to the average control group fractional anisotropy. Values for these calculations were taken from regions that showed a significant group difference. (c) Example of thalamo-cortical tracts, extracted from the structural connectome of a healthy subject with the White Matter Query Language. (d) Significant differences in thalamo-cortical white matter integrity between the TBI and the control group. Each point represents the mean thalamo-cortical values for each subject for fractional anisotropy, mean diffusivity, radial diffusivity and axial diffusivity (from left to right). Although only the values for the global thalamo-cortical fibres are displayed, they are similar for each of the individual thalamic-cortical tracts (thalamo-prefrontal, thalamo-premotor, thalamo-precentral, thalamo-postcentral, thalamo-parietal). 
A
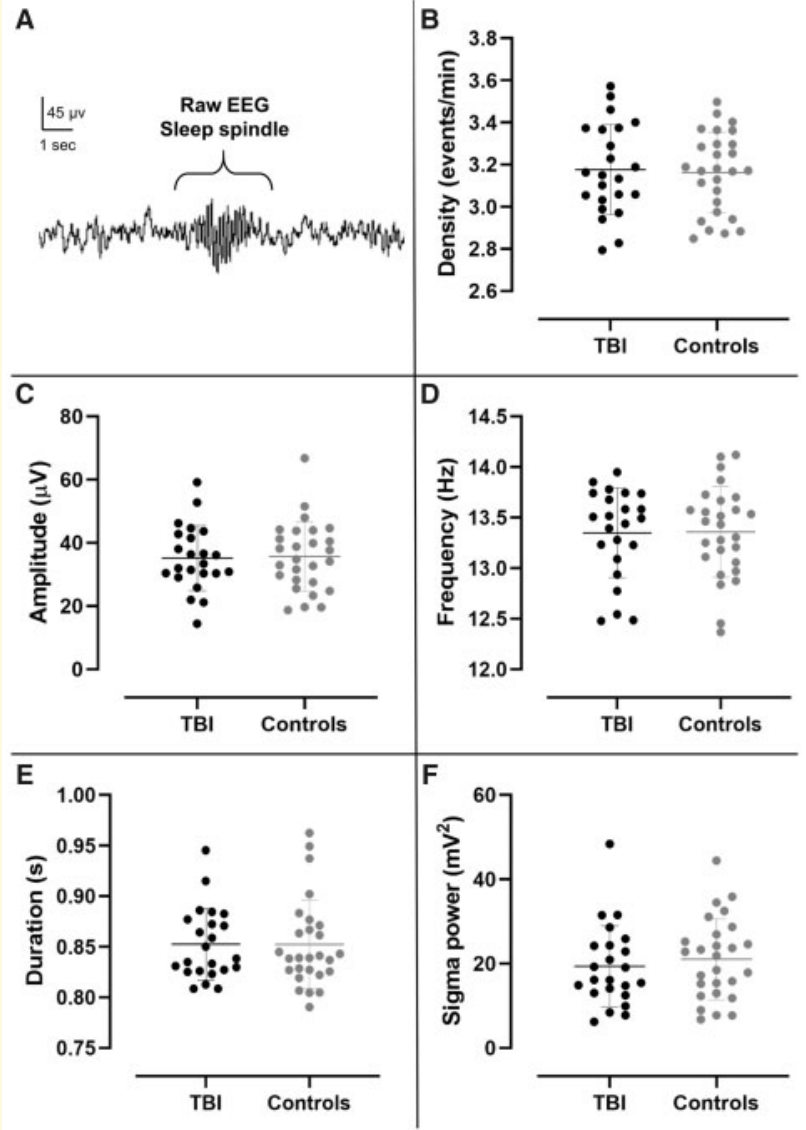

Figure 2 Sleep spindle characteristics in TBI and healthy control groups. Sleep spindles were detected during the N2 and N3 stages of sleep on frontal (F3, F4, Fz), central $(\mathrm{C} 3, \mathrm{C} 4, \mathrm{Cz})$ and parietal (P3, P4, Pz) electrodes for the entire night. (a) Example of a typical sleep spindle, taken from the raw EEG signal of a healthy control subject. (b-f) Sleep spindle characteristics were compared between groups using Student's $t$-tests. The mean of all frontal, central and parietal electrodes is presented on the graphs. No significant difference was found for all characteristics between the TBI group and the healthy control group, for each electrode cluster individually as well as for the mean of all electrodes. Statistical significance was set at $P<0.05$.

amplitude, frequency, duration and sigma-band power for any of the electrode clusters, as well as for the mean of all clusters (Fig. 2). Moreover, in the TBI group, sleep spindles were not associated with markers of TBI severity, most notably the Glasgow Coma Scale score upon hospital admission and the post-traumatic amnesia duration.

\section{Sleep spindles are mostly not associated with white matter damage}

To investigate the association between sleep spindles and white matter damage, we first performed whole-brain
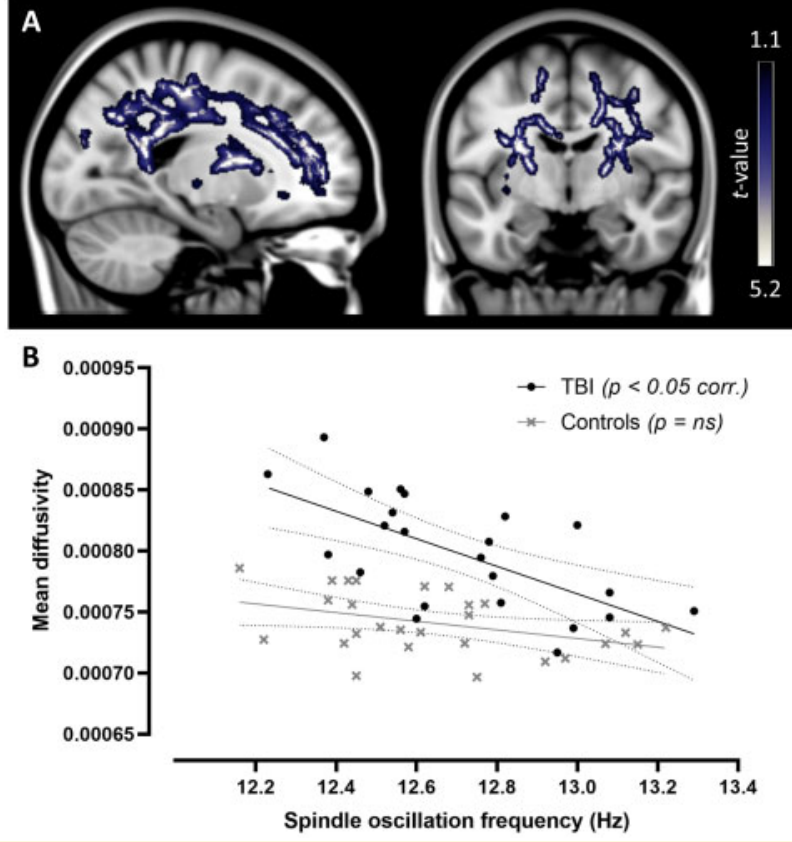

Figure 3 Sleep spindle oscillation frequency and white matter damage. (a) Brain regions (blue-grey) in the TBI group where lower spindle oscillation frequency is correlated with higher mean diffusivity $(r=-0.66$; thresholded at $P<0.05$; corrected for age and multiple comparisons). Significant results are overlaid over the MNII52 TI I mm brain. No significant association was found in the control group. (b) The correlation between the mean value of significant voxels for each subject and the oscillation frequency for the frontal electrode cluster is shown on the graph. Dotted lines represent the $95 \%$ confidence interval.

voxel-wise correlations with Tract-Based Spatial Statistics between spindle characteristics (density, amplitude, frequency, duration and sigma power) in each cluster of electrodes and markers of white matter (fractional anisotropy, mean, axial and radial diffusivity) in TBI and control groups separately. No associations were found between markers of white matter and most spindles' characteristics, except for the TBI group where spindle oscillation frequency was associated with both mean diffusivity and radial diffusivity. More specifically, in TBI subjects, lower spindle frequency was associated with higher diffusivities in voxel clusters spread over the genu, body and splenium of corpus callosum, the anterior, posterior and retrolenticular limbs of internal capsule, the anterior, superior and posterior corona radiata, the posterior thalamic radiation, the sagittal stratum, the external capsule and the superior longitudinal fasciculus (Fig. 3). These associations were more widespread for the frontal cluster of electrodes (22 338-25 887 voxels; $r=0.60-0.66)$, compared with more localized results for the central (541-2168 voxels; $r=0.67-0.75)$ and parietal (1320-3994 voxels; $r=0.68-0.70)$ clusters. Again in the TBI group, spindle duration was associated with both mean and radial diffusivity, but only for the parietal 
cluster of electrodes (3064-10 241 voxels; $r=0.56-0.59$ ): TBI subjects with shorter spindle duration had higher diffusivities in most of the same regions mentioned above. No other associations were found in either TBI or control groups.

To examine more precisely the association between sleep spindles and all individual thalamo-cortical white matter tracts, and how it differed in TBI compared to control groups, we also performed hierarchical linear regressions with spindle characteristics and markers of white matter in thalamo-cortical tracts. Those tracts of interest were extracted with high angular resolution diffusion imaging probabilistic tractography from the wholebrain structural connectomes using all queries presented in Table 1 . We observed that groups had no moderating effect on the association between white matter diffusion properties in the thalamo-cortical tracts and sleep spindle characteristics in any of the three electrode clusters.

\section{Discussion}

In the present study, we tested the hypothesis that damage to cerebral white matter, including the white matter underlying the thalamo-cortical loop, affects sleep spindle generation and morphology. However, we found that sleep spindles were surprisingly resilient to white matter deterioration. In fact, although extensive thalamo-cortical white matter deterioration was evident in the TBI group when quantified from both high angular resolution diffusion imaging probabilistic tractography and a whole-brain voxel-wise approach, sleep spindles did not differ when compared to the healthy control group. Furthermore, sleep spindles were mostly not associated with the degree of white matter deterioration, except that greater white matter damage in the TBI group correlated with lower spindle frequency and duration. Our results highlight the potential resilience of sleep spindle characteristics, and most particularly their density, amplitude and sigma-band power, to white matter deterioration, as they remain unchanged even in a population with chronic extensive white matter damage.

Sleep spindles are a complex network event that relies on a thalamo-cortical loop [see Clawson et al. (2016) for a review]. More specifically, they are generated by the interaction between reticular thalamic neurons and thalamo-cortical neurons, and are often initiated, driven and synchronized by the excitatory feedback of cortico-thalamic neurons. At the outset, reticular thalamic neurons fire in highly synchronous bursts, which causes thalamocortical neurons in the neighbouring thalamic nuclei to send excitatory inputs to their cortico-thalamic targets and to reticular thalamic neurons, setting off the cycle. During the first half of the spindle, the oscillations grow in amplitude with each cycle, thought to be due to the gradually increased activity of cortico-thalamic neurons, further increasing synchrony through the excitatory feedback loop on the thalamus. During the second half of the spindle, all three groups of neurons (cortico-thalamic, thalamo-cortical and reticular thalamic) nearly simultaneously become less rhythmic as the oscillations desynchronize and the spindle amplitude tumbles down, until the spindle is terminated.

Due to the reliance on a cortico-thalamic loop to generate and synchronize the oscillations, we hypothesized that spindle amplitude, density and sigma-band power would be reduced by the extensive damage to white matter tracts. As our results show, they remain unaffected by significant white matter deterioration in participants with moderate to severe TBI. This is, however, mostly consistent with our previous work in which we found that extensive white matter damage did not prevent the expected high cerebral synchrony during sleep (Sanchez et al., 2019). In fact, in our previous study, subjects with more severe white matter damage showed a consistent pattern of exceedingly large and synchronous slow oscillations, which we partly attributed to cerebral disconnection. Indeed, in vivo cat experiments of cortical deafferentation also show that cortical synchrony is chronically enhanced in the undercut hemisphere (Avramescu and Timofeev, 2008; Avramescu et al., 2009; Timofeev et al., 2013). This is likely due to adaptive mechanisms engaged when network activity is acutely decreased after the injury that work to up-regulate network excitability through intrinsic neuronal excitability and the balance of inhibitory and excitatory synapses (Turrigiano et al., 1998, 2011). Considering this timeframe of network excitability, we can infer that spindle amplitude could be acutely decreased following the injury and recover to normal amplitude as the network excitability rises to normal levels. This hypothesis is partly supported by the previous study on eight TBI participants with good outcomes in which spindle amplitude during a 30-min nap increased between 80- and 151-days post-injury, reaching levels comparable to healthy controls (Urakami, 2012). Furthermore, our results also reinforce a recent study on healthy young and old adults which concluded that thalamo-cortical white matter does not explain the agerelated decrease in sleep spindles (Gaudreault et al., 2018). On the other hand, two other studies found an association between reduced white matter integrity and decreased spindle density (Piantoni et al., 2013; Mander et al., 2017). The first, performed on 15 healthy all-male young adults, found that lower axial diffusivity in the left middle temporal lobe with decreased spindle density (Piantoni et al., 2013). The second, which included 20 healthy young adults and 31 healthy older adults, found that higher mean diffusivity in commissural and projecting fibre tracts correlated with decreased fast spindle density in the fourth quartile of the night in one central midline electrode, but not in either frontal or posterior electrodes (Mander et al., 2017). In our healthy control group, sleep spindles were not associated with the interindividual variability in white matter integrity, and do not corroborate these previous reports. These 
discrepancies may be explained by the age disparity between the different control groups, as well as the age-correction applied to all our analyses to control for these effects. These differences also highlight the need for studies to include topographical data when examining sleep oscillations, as they may vary according to the cortical area recorded.

We also found that although spindle duration and oscillation frequency were globally preserved in the TBI group when compared to the healthy control group, both were associated with markers of white matter deterioration in the TBI group only. First, lower spindle duration in posterior electrodes of TBI subjects was associated with more white matter deterioration. This decrease in duration with posterior prominence is very similar to what can be found in ageing (Martin et al., 2013; Rosinvil et al., 2015). Second, we found an association in which TBI subjects with more white matter deterioration to projection and commissural fibres had lower oscillation frequency. Spindles measured on the EEG are network events generated by the back and forth activity along the thalamo-cortical loop (Clawson et al., 2016), and a recent study proposed that the length of the projection fibres determines the oscillation frequency of spindles, when conduction speed is kept constant (Gaudreault et al., 2017). They showed, in healthy adults, that longer fibres were associated with reduced oscillation frequency. Indeed, with longer fibres, the back and forth activity between the thalamus and the cortex becomes slower, and less cycles are achieved before the spindle becomes desynchronized and terminates. However, conduction speed is probably heterogenous in our TBI sample. Both radial diffusivity and mean diffusivity, which were significantly increased along all the thalamo-cortical tracts in the TBI group, are indirect markers of myelin deterioration and showed great variability in this group. Myelin acts as an electrical insulator to achieve saltatory conduction and greatly increases the speed of action potentials (Hartline and Colman, 2007). Consequently, a loss of myelin has been shown to reduce conduction speed and can lead to severe neurological problems (Waxman, 1977; Kutzelnigg et al., 2005; Waxman, 2006; Marion et al., 2018). We show here that the reduced conduction speed potentially present in our TBI sample is associated with decreased oscillation frequency. This supports the model advanced previously (Gaudreault et al., 2017) and suggests that the conduction speed along the thalamo-cortical tracts, in addition to the length of those fibres, can modulate the speed at which the spindles' cycles occur.

This study represents the first to investigate the association of sleep spindles and thalamo-cortical white matter in a human model of severe white matter deterioration. Extensive damage to the white matter was evident across the brain, including in the thalamo-cortical tracts, but we show here that even with such a severe traumatic brain damage, the brain has the capacity to adapt or to withstand alterations in order to conserve normal sleep spindles. Our study included a small proportion of female participants, and while it fully reflects the reality of TBI incidence, it did not allow for stratified sex analyses. It would be interesting for further studies in larger cohorts to investigate the effects of sex while considering factors known to modulate spindles in women, such as menstrual cycles and hormonal contraceptive intake. As this study is also limited by its cross-sectional design, our ability to interpret the underlying mechanisms of this resilience is restricted. However, we know that spindles are essential for cerebral plasticity during sleep (Gais et al., 2002; Schabus et al., 2006; Fogel and Smith, 2011), and for maintaining the integrity of sleep by preventing sleep-interrupting arousals (Naitoh et al., 1982; Dang-Vu et al., 2011). As such, the brain has considerable benefit to maintain normal spindles in the face of severe disruption; their potential restoration over time should be considered crucial in underlying the brain's recovery after a disrupting event (Duclos et al., 2014, 2015), and should be further investigated. Furthermore, this study supports the trait-like properties of spindles, known for their considerable intra-individual stability across nights (De Gennaro et al., 2005; Purcell et al., 2017). We sustain that they are strongly influenced by intrinsic factors other than the brain's white matter, such as the functional connectivity of cortical-thalamic networks and the genetic fingerprint influencing those systems. Ultimately, these results highlight the resilience of sleep spindles, as they remain almost completely undisrupted by extensive white matter deterioration.

\section{Acknowledgements}

For their support in data acquisition, the authors wish to thank Catherine Duclos, Solenne Van der Maren, Caroline D’Aragon, Julien Lauzier, Carollyn Hurst and André Cyr.

\section{Funding}

This study was funded by government granting agencies by grants to N.G. as a principal investigator: Canadian Institutes of Health Research (CIHR MOP 115172) and Fonds de Recherche Santé-Québec (FRQS \#34851). E.S. and A.-A.B. received scholarships from the CIHR and FRQS. N.G. received a salary award from the FRQS. J.C. received a salary award from the CIHR. M.D. holds a Universite de Sherbrooke Research Chair in Neuroinformatics.

\section{Competing interests}

The authors declare no competing interests.

\section{References}

Avramescu S, Nita DA, Timofeev I. Neocortical post-traumatic epileptogenesis is associated with loss of GABAergic neurons. J Neurotrauma 2009; 26: 799-812.

Avramescu S, Timofeev I. Synaptic strength modulation after cortical trauma: a role in epileptogenesis. J Neurosci 2008; 28: 6760-72. 
Clawson BC, Durkin J, Aton SJ. Form and function of sleep spindles across the lifespan. Neural Plast 2016; 2016: 1-16.

Dang-Vu TT, Bonjean M, Schabus M, Boly M, Darsaud A, Desseilles $\mathrm{M}$, et al. Interplay between spontaneous and induced brain activity during human non-rapid eye movement sleep. Proc Natl Acad Sci U S A 2011; 108: 15438-43.

De Gennaro L, Ferrara M, Vecchio F, Curcio G, Bertini M. An electroencephalographic fingerprint of human sleep. NeuroImage 2005; 26 : 114-22.

Descoteaux M, Deriche R, Knosche TR, Anwander A. Deterministic and probabilistic tractography based on complex fibre orientation distributions. IEEE Trans Med Imaging 2009; 28: 269-86.

Duclos C, Beauregard MP, Bottari C, Ouellet MC, Gosselin N. The impact of poor sleep on cognition and activities of daily living after traumatic brain injury: a review. Aust Occup Ther J 2015; 62: 2-12.

Duclos C, Dumont M, Blais H, Paquet J, Laflamme E, de Beaumont L, et al. Rest-activity cycle disturbances in the acute phase of moderate to severe traumatic brain injury. Neurorehabil Neural Repair 2014; 28: 472-82.

Fogel SM, Nader R, Cote KA, Smith CT. Sleep spindles and learning potential. Behav Neurosci 2007; 121: 1-10.

Fogel SM, Smith CT. The function of the sleep spindle: a physiological index of intelligence and a mechanism for sleep-dependent memory consolidation. Neurosci Biobehav Rev 2011; 35: 1154-65.

Gais S, Molle M, Helms K, Born J. Learning-dependent increases in sleep spindle density. J Neurosci 2002; 22: 6830-4.

Garyfallidis E, Brett M, Amirbekian B, Rokem A, van der Walt S, Descoteaux M, et al.; Dipy Contributors. Dipy, a library for the analysis of diffusion MRI data. Front Neuroinform 2014; 8: 8 .

Gaudreault PO, Carrier J, Descoteaux M, Deslauriers-Gauthier S. Is the length of the white matter fiber bundles underlying the thalamocortical loop associated with sleep spindles? A preliminary study. Proc Intl Soc Mag Reson Med 2017; 25. Available at: http://index smart.mirasmart.com/ISMRM2017/PDFfiles/4704.html.

Gaudreault PO, Gosselin N, Lafortune M, Deslauriers-Gauthier S, Martin N, Bouchard M, et al. The association between white matter and sleep spindles differs in young and older individuals. Sleep 2018; 41: zsy113. doi: 10.1093/sleep/zsy113.

Gennarelli TA, Thibault LE, Adams JH, Graham DI, Thompson CJ, Marcincin RP. Diffuse axonal injury and traumatic coma in the primate. Ann Neurol 1982; 12: 564-74.

Haberg AK, Olsen A, Moen KG, Schirmer-Mikalsen K, Visser E, Finnanger TG, et al. White matter microstructure in chronic moderate-to-severe traumatic brain injury: impact of acute-phase injuryrelated variables and associations with outcome measures. J Neurosci Res 2015; 93: 1109-26.

Hartline DK, Colman DR. Rapid conduction and the evolution of giant axons and myelinated fibers. Curr Biol 2007; 17: R29-35.

Iber C, Ancoli-Israel S, Quan S, The AASM manual for the scoring of sleep and associated viii events: rules, terminology, and technical specifications. Westchester, IL: American Academy of Sleep Medicine; 2007.

Johnson VE, Stewart W, Smith DH. Axonal pathology in traumatic brain injury. Exp Neurol 2013; 246: 35-43.

Kennedy MR, Wozniak JR, Muetzel RL, Mueller BA, Chiou HH, Pantekoek $\mathrm{K}$, et al. White matter and neurocognitive changes in adults with chronic traumatic brain injury. J Int Neuropsychol Soc 2009; 15: 130-6.

Kraus MF, Susmaras T, Caughlin BP, Walker CJ, Sweeney JA, Little DM. White matter integrity and cognition in chronic traumatic brain injury: a diffusion tensor imaging study. Brain 2007; 130: 2508-19.

Kumar R, Husain M, Gupta RK, Hasan KM, Haris M, Agarwal AK, et al. Serial changes in the white matter diffusion tensor imaging metrics in moderate traumatic brain injury and correlation with neuro-cognitive function. J Neurotrauma 2009; 26: 481-95.

Kutzelnigg A, Lucchinetti CF, Stadelmann C, Bruck W, Rauschka H, Bergmann $\mathrm{M}$, et al. Cortical demyelination and diffuse white matter injury in multiple sclerosis. Brain 2005; 128: 2705-12.
Mander BA, Zhu AH, Lindquist JR, Villeneuve S, Rao V, Lu B, et al. White matter structure in older adults moderates the benefit of sleep spindles on motor memory consolidation. J Neurosci 2017; 37: 11675-87.

Marion CM, Radomski KL, Cramer NP, Galdzicki Z, Armstrong RC. Experimental traumatic brain injury identifies distinct early and late phase axonal conduction deficits of white matter pathophysiology, and reveals intervening recovery. J Neurosci 2018; 38: 8723-36.

Martin N, Lafortune M, Godbout J, Barakat M, Robillard R, Poirier $\mathrm{G}$, et al. Topography of age-related changes in sleep spindles. Neurobiol Aging 2013; 34: 468-76.

Menon DK, Schwab K, Wright DW, Maas AI. Position statement: definition of traumatic brain injury. Arch Phys Med Rehabil 2010; 91: 1637-40.

Mori S, Oishi K, Jiang H, Jiang L, Li X, Akhter K, et al. Stereotaxic white matter atlas based on diffusion tensor imaging in an ICBM template. NeuroImage 2008; 40: 570-82.

Naitoh P, Antony-Baas V, Muzet A, Ehrhart J. Dynamic relation of sleep spindles and K-complexes to spontaneous phasic arousal in sleeping human subjects. Sleep 1982; 5: 58-72.

Nakase-Richardson R, Sepehri A, Sherer M, Yablon SA, Evans C, Mani T. Classification schema of posttraumatic amnesia durationbased injury severity relative to 1-year outcome: analysis of individuals with moderate and severe traumatic brain injury. Arch Phys Med Rehabil 2009; 90: 17-9.

Piantoni G, Poil SS, Linkenkaer-Hansen K, Verweij IM, Ramautar JR, Van Someren EJ, et al. Individual differences in white matter diffusion affect sleep oscillations. J Neurosci 2013; 33: 227-33.

Pitkonen M, Abo-Ramadan U, Marinkovic I, Pedrono E, Hasan KM, Strbian D, et al. Long-term evolution of diffusion tensor indices after temporary experimental ischemic stroke in rats. Brain Res 2012; 1445: 103-10.

Purcell SM, Manoach DS, Demanuele C, Cade BE, Mariani S, Cox R, et al. Characterizing sleep spindles in 11,630 individuals from the National Sleep Research Resource. Nat Commun 2017; 8: 15930.

Rosinvil T, Lafortune M, Sekerovic Z, Bouchard M, Dubé J, LatulipeLoiselle A, et al. Age-related changes in sleep spindles characteristics during daytime recovery following a 25 -hour sleep deprivation. Front Hum Neurosci 2015; 9: 323.

Sanchez E, El-Khatib H, Arbour C, Bedetti C, Blais H, Marcotte K, et al. Brain white matter damage and its association with neuronal synchrony during sleep. Brain 2019; 142: 674-87.

Schabus M, Dang-Vu TT, Heib DP, Boly M, Desseilles M, Vandewalle G, et al. The fate of incoming stimuli during NREM sleep is determined by spindles and the phase of the slow oscillation. Front Neur 2012; 3: 40.

Schabus M, Hodlmoser K, Gruber G, Sauter C, Anderer P, Klosch G, et al. Sleep spindle-related activity in the human EEG and its relation to general cognitive and learning abilities. Eur J Neurosci 2006; 23: 1738-46.

Smith DH, Hicks R, Povlishock JT. Therapy development for diffuse axonal injury. J Neurotrauma 2013; 30: 307-23.

Smith SM, Jenkinson M, Johansen-Berg H, Rueckert D, Nichols TE, Mackay CE, et al. Tract-based spatial statistics: voxelwise analysis of multi-subject diffusion data. NeuroImage 2006; 31: 1487-505.

Smith SM, Jenkinson M, Woolrich MW, Beckmann CF, Behrens TE, Johansen-Berg $\mathrm{H}$, et al. Advances in functional and structural MR image analysis and implementation as FSL. NeuroImage 2004; 23 : S208-19.

Teasdale G, Jennett B. Assessment of coma and impaired consciousness. A practical scale. Lancet 1974; 304: 81-4.

Timofeev I, Sejnowski TJ, Bazhenov M, Chauvette S, Grand LB. Age dependency of trauma-induced neocortical epileptogenesis. Front Cell Neurosci 2013; 7: 154.

Turrigiano G. Too many cooks? Intrinsic and synaptic homeostatic mechanisms in cortical circuit refinement. Annu Rev Neurosci 2011; 34: 89-103.

Turrigiano GG, Leslie KR, Desai NS, Rutherford LC, Nelson SB. Activity-dependent scaling of quantal amplitude in neocortical neurons. Nature 1998; 391: 892-6. 
Urakami Y. Relationship between, sleep spindles and clinical recovery in patients with traumatic brain injury: a simultaneous EEG and MEG study. Clin EEG Neurosci 2012; 43: 39-47.

Vien C, Bore A, Boutin A, Pinsard B, Carrier J, Doyon J, et al. Thalamo-cortical white matter underlies motor memory consolidation via modulation of sleep spindles in young and older adults. Neuroscience 2019; 402: 104-15.

Wassermann D, Makris N, Rathi Y, Shenton M, Kikinis R, Kubicki $\mathrm{M}$, et al. On describing human white matter anatomy: the White Matter Query Language. Med Image Comput Comput Assist Interv 2013; 16: 647-54.
Wassermann D, Makris N, Rathi Y, Shenton M, Kikinis R, Kubicki $\mathrm{M}$, et al. The White Matter Query Language: a novel approach for describing human white matter anatomy. Brain Struct Funct 2016; 221: 4705-21.

Waxman SG. Conduction in myelinated, unmyelinated, and demyelinated fibers. Arch Neurol 1977; 34: 585-9.

Waxman SG. Axonal conduction and injury in multiple sclerosis: the role of sodium channels. Nat Rev Neurosci 2006; 7: 932-41.

Winkler AM, Ridgway GR, Webster MA, Smith SM, Nichols TE. Permutation inference for the general linear model. NeuroImage 2014; 92: 381-97. 